\title{
Occupational exposures and utilisation of HIV post- exposure prophylaxis amongst health workers of three hospitals in Tanzania's Lake Zone
}

\begin{abstract}
Authors:
Rogatus Kabyemera ${ }^{1}$

Albino Kalolo ${ }^{2}$

Geethika Fernando ${ }^{3}$

Susan Bergson ${ }^{3}$

\section{Affiliations:}

${ }^{1}$ Department of Paediatrics and Child Health, Bugando Medical Centre, Tanzania

${ }^{2}$ Department of Community Health, St. Francis University College of Health and Allied

Sciences, Tanzania
\end{abstract}

${ }^{3}$ Monitoring and Evaluation, AmeriCares, United States

Correspondence to:

Rogatus Kabyemera

Email:

mrkabyemera@gmail.com

Postal address:

PO Box 1370, Mwanza, Tanzania

Dates:

Received: 08 May 2015

Accepted: 11 Sept. 2015

Published: 12 Oct. 2015

How to cite this article: Kabyemera, R., Kalolo, A., Fernando, G. \& Bergson, S., 2015, 'Occupational exposures and utilisation of HIV post-exposure prophylaxis amongst health workers of three hospitals in Tanzania's Lake Zone', African Evaluation Journal 3(2), Art. \#152, 8 pages. http://dx.doi. org/10.4102/aej.v3i2.152

\section{Copyright:}

(C) 2014. The Authors. Licensee: AOSIS OpenJournals. This work is licensed under the Creative Commons Attribution License.
Occupational exposures predispose health workers (HWs) to a risk of HIV infection. When properly used, HIV Post Exposure Prophylaxis (PEP) can significantly reduce this risk. The purpose of this study was to determine the extent and types of occupational exposures, availability of PEP guidelines, and utilisation of PEP amongst HWs. A cross-sectional mixed methods baseline study was conducted between March and May 2014 using a structured questionnaire administered to $236 \mathrm{HWs}$ from Shinyanga, Sengerema and Musoma hospitals in Tanzania. Relationships between variables were determined using the chi-square test. Qualitative data was gathered during interviews with key informants and walkthrough observations in the hospital wards, and analysed using the framework method. Majority of respondents were nurses (53\%), over 40 years old (61\%) with more than 20 years $(40 \%)$ of hospital experience. Needle stick and sharp injuries were experienced by $31 \%$ of respondents whilst $26 \%$ experienced splashes. Only $28 \%$ of splashes were reported compared to $80 \%$ and $68 \%$ of needle sticks and sharp injuries, respectively. Those who experienced needle sticks were more likely to report the incident $(p<0.001)$, receive testing $(p<0.003)$ or PEP $(p=$ $0.005)$. Although $66 \%$ reported the availability of PEP guidelines, only $39 \%$ of exposed HWs received PEP. Occupational exposures are common in these hospitals. HWs under-report and suboptimally use PEP services. Health worker safety programmes should establish functional systems for reporting and adherence to PEP procedures. Future research should establish factors that determine compliance with PEP procedures.

\section{Introduction}

\section{Background and literature review}

Health workers (HW) are potentially exposed to Human Immunodeficiency Virus (HIV) infection at their workplace as a result of exposures to potentially infectious materials in the course of performing their duties (Cardo et al. 1997; Kuhar et al. 2013; Lee 2009; Sagoe-Moses et al. 2001). Evidence shows that occupational injuries account for an average of $4.4 \%$ (ranging from $0.8 \%$ to 18.5\%) of all HIV infections amongst HWs (Prüss-Üstün \& Rapiti 2003; Prüss-Üstün, Rapiti \& Hutin 2005). The estimated risk of HIV transmission ranges between $0.09 \%$ and $0.3 \%$ when one is exposed to infected body fluids (splashes) and percutaneous injuries (needle sticks and sharp injuries) respectively (Gold \& Tomkins 2005).

The World Health Organization (WHO) estimates that 3 million percutaneous exposures amongst HWs occur every year resulting in 200 to $5000 \mathrm{HIV}$ infections, with over $90 \%$ occurring in low-resource countries (Varghese, Abraham \& Mathai 2003) where HWs suffer two to four needle-stick injuries per year (Prüss-Üstün et al. 2005).

Despite major improvements in the fight against HIV infection, HWs in developing countries are far more at risk of occupational HIV infection than their counterparts in developed countries (Lee 2009). The observed differences might be a result of the challenges faced by developing countries in provision of health care. These challenges include crowded hospitals, high patient load per HW, inadequate personal protective equipment (PPE), lack of sharps containers, limited knowledge of risks and utilisation of Post Exposure Prophylaxis (PEP), low adherence to universal precautions (UP), and high prevalence of patients with blood borne infections (Prüss-Üstün \& Rapiti 2003; Sultan, Benn \& Waters 2014). Sub-Saharan Africa has both the highest prevalence of HIV infection and the highest incidence of occupational exposures which, on average, was reported to be 2.10 injuries per HW per year (Lee 2009; Prüss-Üstün \& Rapiti 2003; Prüss-Üstün et al. 2005). 
To reduce occupational HIV infection, WHO recommends the use of PEP, defined as a short-term antiretroviral treatment taken in order to reduce the likelihood of HIV infection after potential exposure (WHO 2015). When PEP is properly administered shortly after exposure, the risk of transmission of HIV infection as a result of the exposure is reduced by $81 \%$ (Birdsall et al. 2004; Sari et al. 2011).

Reporting of exposures is important in order to access PEP services. However, recent studies show insufficient reporting of occupational exposures. A study done in Switzerland found that up to $58.6 \%$ of the exposures were not reported leading to insufficient use of PEP services (Voide et al. 2012). Researchers have identified reasons for not using HIV PEP services after exposure including: fear of reprimand, uncertainty regarding the confidentiality of the results, unfamiliarity with the existence of a protocol for reporting and dealing with occupational exposure as well as a lack of support and encouragement to report (Kuhar et al. 2013; Tebeje \& Hailu 2010). In addition, belief that the exposure was not significant and being too busy were reasons for underreporting (Kessler et al. 2011).

Tanzania is one of the sub-Sahara African countries that has a high burden of HIV and health work force crisis (Kwesigabo et al. 2012). The national HIV prevalence is 5.1\% with variations across regions (TACAIDS 2013) and human resource for health stands at 65\% (Kwesigabo et al. 2012; TACAIDS 2013). Since 2004, the Ministry of Health and Social Welfare (MOHSW) in Tanzania has been implementing a national PEP programme as part of comprehensive HIV care, treatment and prevention services (NACP 2012).

With increased attention to improving health workplace safety and prevention of occupational related diseases for HWs, there is a need to expand the knowledge base on the role of contextual factors associated with prevalence of occupational exposures, reporting of injuries and availability and use of PEP amongst HWs. In Tanzania, there are very few studies that have explored the problem of occupational exposures and use of PEP using multisite mixed methods design.

Efforts to improve health workplace safety in Tanzania are facilitated in part through the Health Worker Safety (HWS) programme that started in 2009 at Bugando Medical Centre (BMC) in Mwanza region with support from AmeriCares. In 2014, the programme was proposed for extension to Shinyanga, Sengerema and Musoma hospitals in Tanzania's lake zone. Apart from the other components of HWS, the programme aims to reduce occupational exposures, improve reporting of occupational injuries, and PEP use amongst HWs in the three beneficiary hospitals.

As part of the larger HWS programme baseline evaluation study that aimed to establish baseline indicators and possible counterfactuals, this article presents a component of the study that aimed at establishing the reality with regards to occupational injuries and utilisation of PEP services. More specifically, this study aimed to:

- Determine the extent of and types of occupational exposures.

- Describe the availability of PEP guidelines and services.

- Describe the utilisation of PEP services amongst HWs.

\section{Research method and design}

In this section we present the settings of the study, the study design, the data collection methods and the analytical approach for the collected data.

\section{Setting}

In order to learn about the differences and similarities between regional and district hospitals, public and publicprivate hospitals, this study was conducted in Shinyanga Regional Referral Hospital (SRRH), Sengerema Designated District Hospital (SDDH) and Musoma Regional Referral Hospital (MRRH) in Tanzania's lake zone.

Tanzania's lake zone comprises six regions found around Lake Victoria, including: Mwanza, Shinyanga, Kagera, Geita, Simiyu and Mara. SRRH is a secondary level government hospital located in Shinyanga region. It has a 304 bed capacity, 338 HWs (29\% less than optimal staffing levels) and an annual budget of approximately 6182424 US dollars. $\mathrm{SDDH}$ is a designated hospital for Sengerema district council, owned by the Catholic Diocese of Geita, and located in the Mwanza region. It has a bed capacity of 301 and has 275 HWs ( $11 \%$ less than optimal staffing levels) and a budget of approximately 1793360 US dollars. MRRH is a government owned hospital with a bed capacity of 300 and is staffed with 366 HWs, which is $23 \%$ less than optimal staffing levels. The hospital has a budget of approximately 2606060 US dollars.

\section{Design}

This evaluation employed a cross-sectional concurrent mixed methods design (Creswell \& Plano Clark 2011; Creswell et al. 2011). The cross-sectional component refers to the one specific point in time of data collection and analysis, whereas the mixed methods component refers to the combination of quantitative and qualitative methods of data collection and analysis. Taking a pragmatic stance, we adopted a mixed methods approach in an effort to obtain a comprehensive reality at baseline as one method alone might not be sufficient given the complexity of the studied phenomenon (Feilzer 2010). This study was conducted from March to May 2014 amongst HWs in Shinyanga, Sengerema and Musoma hospitals.

\section{Procedure}

HWs employed by the three hospitals were the target population for this study.

In the quantitative component of the study, a sample for the knowledge, attitude and practice (KAP) survey was 
obtained using a convenient sampling technique whereby all HWs present at morning and afternoon shifts during the data collection period were included in the study upon informed consent. In Shinyanga we recruited 100 out of 338 staff $(29.6 \%)$, whereas in Sengerema, 60 HWs out of $275(21.8 \%)$ staff were recruited in the study. We recruited 104 participants out of 366 staff (28.4\%) in Musoma. Participants were HWs involved in handling patients or infectious materials from patients and included medical doctors, nurses, laboratory personnel, pharmacists and other support staff.

The KAP survey captured sociodemographic characteristics on HWs and factors influencing responses such as the availability of HWS-related materials to enhance practices. The KAP survey questionnaire was developed as recommended by previous studies on occupational exposures (Geer et al. 2006).

In the qualitative component of the study, respondents for in-depth interviews (individual and group) were selected using a purposive sampling technique based on their presumed knowledge about the subject under study such as individuals who occupy certain positions at the hospital and those who work directly with patients or patient materials. The list of participants who were recruited for interviews by virtue of their positions in each hospital included: medical officer in charge, hospital secretary, hospital matron, hospital pharmacist, and heads of selected clinical departments (surgery, obstetrics and gynaecology and paediatrics). Amongst those who work directly with patients or patient materials and were interviewed for the study were doctors, nurses, laboratory workers, medical attendants and waste handlers. In-depth interviews were conducted with a minimum of 10 individuals per hospital.

Members of the Hospital Management Team (HMT) in each of the three hospitals were invited for a participatory in-depth interview. Facilitated by the evaluators (RK and AK), the interview mimicked the usual HMT meetings at the hospital whereby participants were encouraged to contribute to the interview questions in a participatory manner. Participatory group in-depth interviews were conducted with at most 12 HMT members of each hospital.

In both the individual and group in-depth interviews, we asked questions related to general knowledge, attitudes and practices in the context of occupational injuries and use of PEP at each hospital. Furthermore, specific questions were addressed to individual participants depending on their roles in occupational injuries and PEP issues.

We also employed walkthrough observations to purposively selected hospital sections depending on their relevance to occupational injuries and use of PEP. Such sections included: emergency departments, surgical wards, labour wards and operating theatres, hospital laboratories and infectious waste disposal sites. An observation checklist was used to assess the presence or absence of occupational health protocols, materials and tools such as national guidelines, HIV test kits, antiretroviral drugs (ARVs) and PEP reporting forms. The respective departments or wards were unaware of the walkthrough observation before it was conducted.

Data collection was done by research assistants selected based on their level of academic qualifications (mostly bachelor and masters in social science disciplines such as sociology and public health) and experience in conducting both quantitative and qualitative research. The research assistants were trained and the instruments pre-tested prior to the start of data collection. The tools were translated from English to Kiswahili (the national language of Tanzania) to facilitate understanding by the HWs.

Measures of occupational injuries (number, type and reporting status), availability and use of PEP as dependent variables, were based on participants' responses to the binary choice questions ('yes' or 'no') from the KAP questionnaire. The expected responses were ' $1=$ yes' $^{\prime}$ and '2 = No'. Independent variables included participants' hospital location, job role, and length of time at institution and ward or department. We defined occupational injuries as a percutaneous injury (needle sticks or a cut with a sharp object) or contact of mucous membrane or non-intact skin with body fluids (splash). We distinguished needle stick injuries (needle prick) from sharp injuries (injury from other sharp objects) in order to understand the burden of each of the causes. Utilisation of PEP was defined as immediate reporting and (when appropriate) use of antiretroviral drugs (ARVs) administered through the respondent's place of employment following exposure to potentially infectious body fluids in order to reduce the risk of HIV transmission. We hypothesised that occupational injury, their frequency as well as reporting and use of PEP would be associated with job role. At the time of the baseline evaluation, none of the three hospitals had a complete registry to document occupational injuries or to accurately track administration of PEP.

\section{Analyses}

Collected data was checked for consistency, accuracy and completeness during and after data collection as well as during data entry exercises.

Quantitative data was entered into electronic databases using an agreed coding system for each question from the KAP survey. MS Excel 2010 and STATA 13 were used for entering and cleaning quantitative data. Data entry screens were fixed with consistency and completeness checks. Quantitative data was analysed using STATA version 13, applying both descriptive and detailed analysis techniques to determine the relationship between variables.

Although we collected data from 264 HWs, for purposes of this analysis, only doctors, nurses and medical attendants 
were included $(n=236)$. Motivated by our wish to proceed to bivariate analysis, initial analysis revealed that other job roles $(n=28)$ reported small numbers of exposures not suitable for chi-square analysis.

Descriptive statistics on sociodemographic characteristics, occupational injuries, wards or departments with PEP medicines, HWs who reported injuries and used ARVs for PEP as well as reasons for not using ARVs for PEP was performed. When applicable, statistical tests and cross tabulations were carried out in order to determine relationships between variables.

Qualitative data analysis was guided by the Framework method (Gale et al. 2013) and following its six-stage approach, namely:

- Transcribing the collected data.

- Familiarisation with the transcript.

- Coding.

- Developing a working analytical framework.

- Applying the analytical framework.

- Charting the data into framework matrix.

- Interpreting the data.

Audio clips from recorders were listened to and compared with field notes (interviews and observations) and then complete verbatim transcripts was produced. Coding was done by using both inductive and deductive approaches, which is also in line with the framework approach. Codes were shared between the authors for cross-checking as well as quotes that were relevant for emerging themes. In developing the framework matrix, quotes were attached to each code. NVivo software (QSR-International) assisted in the qualitative data analysis.

\section{Ethical considerations}

Ethical clearance was provided by the BMC Institutional Review Board (IRB) in Tanzania. Once permission from Regional and district authorities was granted, participating hospital authorities were asked for informed consent to permit HWs to take part in the study. HWs were asked for consent after being informed about the purpose of the study, the possible benefits of the study, confidentiality, and that participation was completely voluntary. Furthermore, details of the information sheet were stated verbally by the research assistants before distributing the questionnaires or conducting the interview with the HWs.

\section{Results}

The findings of this study are presented according to the three objectives of the study. The first part however, describes the participants' characteristics.

\section{Participants' characteristics}

The majority of respondents were nurses $(53 \%)$, over 40 years $(61 \%)$, from maternity wards $(20 \%)$ or the outpatient
TABLE 1: Baseline characteristics of health workers included in analysis.

\begin{tabular}{lc}
\hline Characteristic & $\boldsymbol{n}(\mathbf{\%})$ \\
\hline Mean age $(\boldsymbol{N}=\mathbf{2 3 6})$ & 43.1 \\
Age range (years) & $20-69$ \\
Age groups & \\
$20-30$ & $35(15)$ \\
$31-40$ & $55(23)$ \\
$41-50$ & $86(37)$ \\
$51-60$ & $57(24)$ \\
$60+$ & $3(1)$ \\
Location ( $N=236)$ & \\
Musoma & $92(39)$ \\
Sengerema & $53(23)$ \\
Shinyanga & $91(38)$ \\
Job Title $(\boldsymbol{N}=\mathbf{2 3 6})$ & \\
Physicians & $28(11)$ \\
Nurses & $124(53)$ \\
Medical attendants & $84(36)$ \\
Department or ward $(\boldsymbol{N}=\mathbf{2 3 6})$ & \\
Female wards & $31(13)$ \\
Labour or maternity ward & $47(20)$ \\
Male wards & $38(16)$ \\
OPD & $43(18)$ \\
Other (including infectious wards) & $31(13)$ \\
Paediatric & $26(11)$ \\
Wheatre & $18(8)$ \\
Length of employment $(\boldsymbol{N}=\mathbf{2 3 6})$ & $60(26)$ \\
0-9 years & $95(40)$ \\
\hline 20 years &
\end{tabular}

department (18\%). In addition, most of the respondents (95 [40\%]) were employed at their respective hospitals for more than 20 years (Table 1 ).

\section{Prevalence of occupational exposures and injury reporting between job roles}

A total of $35 \mathrm{HWs}(15 \%)$ suffered one or more needle-stick injuries (NSI) during a six-month period. However, amongst physicians, $25 \%$ suffered one or more NSIs in the past 6 months closely followed by medical attendants (17\%) (Table 2). Nurses showed the lowest within group prevalence (11\%). However, many of the nurses who experienced a NSI reported on their injury $(93 \%)$ compared to physicians $(86 \%)$ and medical attendants (57\%). No significant association was shown between job role and experiencing the needle-stick injury. A similar trend was shown in sharps injuries with prevalence greatest amongst physicians (29\%). Similar to NSIs, reporting of sharps injuries was lowest amongst medical attendants $(40 \%)$, with reporting significantly associated with job role $\left(\chi^{2}=3.75\right.$, $p=0.004)$. Amongst all exposure types, splash exposures were the most common with $46(20 \%)$ cases of splash injuries during the six-month period. Suffering a splash injury was significantly different between the three job roles $\left(\chi^{2}=6.1\right.$, $p=0.047)$. Not surprisingly, physicians $(36 \%)$ tend to get splashed more than other HWs. However, the proportion of medical attendants suffering all three types of exposures was comparatively higher than that of nurses (Table 2). 
TABLE 2: Prevalence of reported injuries amongst respondents by job role.

\begin{tabular}{|c|c|c|c|c|c|c|}
\hline Injuries & Physicians & Nurses & Medical attendants & Total & $\chi^{2}$ & $P$-value \\
\hline Needle stick injuries (in the past 6 months) & $(n=28)$ & $(n=124)$ & $(n=84)$ & $(N=236)$ & \multirow{4}{*}{3.75} & \\
\hline No injuries $n(\%)$ & $21(75)$ & $110(89)$ & $70(83)$ & $201(85)$ & & \multirow{2}{*}{0.153} \\
\hline 1 or more injuries $n(\%)$ & $7(25)$ & $14(11)$ & $14(17)$ & $35(15)$ & & \\
\hline Reported injuries (Fisher's Exact) $n(\mathbf{\%})$ & $6(86)$ & $13(93)$ & $8(57)$ & $28(80)$ & & 0.099 \\
\hline Sharps injuries (in the past 6 months) & $(n=28)$ & $(n=124)$ & $(n=84)$ & $(N=236)$ & \multirow{4}{*}{4.89} & \multirow{3}{*}{0.086} \\
\hline No injuries $n(\%)$ & $20(71)$ & $109(88)$ & $69(82)$ & $198(84)$ & & \\
\hline 1 or more injuries $n(\%)$ & $8(29)$ & $15(12)$ & $15(18)$ & $38(16)$ & & \\
\hline Reported injuries (Fisher's Exact) $n(\%)$ & $6(75)$ & $14(93)$ & $6(40)$ & $26(68)$ & & 0.004 \\
\hline Splashes (in the past 6 months) & $(n=28)$ & $(n=124)$ & $(n=84)$ & $(N=236)$ & \multirow{4}{*}{6.1} & \multirow{3}{*}{0.047} \\
\hline No injuries $n(\%)$ & $18(64)$ & $105(85)$ & $67(80)$ & $190(80)$ & & \\
\hline 1 or more injuries $n(\%)$ & $10(36)$ & $19(15)$ & $17(20)$ & $46(20)$ & & \\
\hline Reported injuries (Fisher's Exact) $n(\%)$ & $2(20)$ & $2(11)$ & $5(29)$ & $13(28)$ & & 0.35 \\
\hline
\end{tabular}

TABLE 3: Reporting, counselling, testing and receipt of HIV post exposure prophylaxis after an injury or exposure.

\begin{tabular}{|c|c|c|c|c|c|}
\hline Post-exposure action & Needle-stick injuries & Sharps & Splashes & Total & $\begin{array}{c}\text { Fisher's Exact } \\
\text { ( } p \text {-value) }\end{array}$ \\
\hline Reporting of injuries or exposure & $(n=35)$ & $(n=38)$ & $(n=46)$ & $(N=119)$ & \multirow{3}{*}{$<0.001$} \\
\hline Yes $n(\%)$ & $28(80)$ & $26(68)$ & $13(28)$ & $67(56)$ & \\
\hline No $n(\%)$ & $7(20)$ & $12(32)$ & $33(72)$ & $52(43)$ & \\
\hline Offered counselling after injury or exposure & $(n=28)$ & $(n=26)$ & $(n=13)$ & $(N=67)$ & \multirow{3}{*}{0.246} \\
\hline Yes $n(\%)$ & $12(43)$ & $6(23)$ & $3(23)$ & $21(33)$ & \\
\hline No $n(\%)$ & $16(57)$ & $20(77)$ & $10(77)$ & $46(67)$ & \\
\hline Offered disease testing after injury or exposure & $(n=28)$ & $(n=26)$ & $(n=13)$ & $(N=67)$ & \multirow{3}{*}{0.003} \\
\hline Yes $n(\%)$ & $23(82)$ & $10(39)$ & $8(61)$ & $41(61)$ & \\
\hline No $n(\%)$ & $5(18)$ & $16(61)$ & 5 (39) & $26(39)$ & \\
\hline Received PEP & $(n=28)$ & $(n=26)$ & $(n=13)$ & $(N=67)$ & \multirow{3}{*}{0.005} \\
\hline Yes $n(\%)$ & $17(61)$ & $5(19)$ & $4(31)$ & $26(39)$ & \\
\hline No $n(\%)$ & $11(39)$ & $21(81)$ & $9(69)$ & $41(61)$ & \\
\hline
\end{tabular}

PEP, post exposure prophylaxis.

Respondents to in-depth interviews reported the presence of occupational injuries amongst HWs, those handling surgical procedures having more incidences of injuries. Despite reported high incidences of exposures, many of the respondents noted barriers to reporting occupational injuries amongst HWs, including: self-evaluation of the nature of injury, reluctance to receive HIV results and unavailability of counsellors when needed. Some participants also reported that they have been exposed to injuries, reported, were tested for HIV and received PEP:

'Yes, I was injured and I reported it to my supervisors. After that I was offered a HIV test ... and received PEP after a negative test.' (Medical attendant)

\section{Reporting, counselling, testing, and receipt of post exposure prophylaxis after an injury or exposure}

Splashes (28\%) were relatively under-reported compared to NSIs $(80 \%)$ and sharps $(68 \%)(p<0.001)$. A third of HWs were offered counselling (33\%) after reporting an injury, more than half were offered disease testing (61\%) and slightly more than a third received PEP (39\%). Those who experienced needle sticks injuries were more likely to be offered counselling $(43 \%)$, receive testing $(82 \%)(p=0.003)$ and receive PEP $(61 \%)$ $(p=0.005)$ compared to other exposure types. Although splash injuries were under-reported compared to sharps injuries, the proportion who received PEP after a splash (39\%) was greater than those who suffered sharps injuries $(31 \%)(p=0.005)$ (Table 3).
Qualitative data indicates that despite the availability of information about the need to report injuries after occupational exposure, HWs decision to report injuries was seen to be influenced by complexities related to HIV stigma:

'All HWs know that they have to report if they sustain needlestick injury but choose not to report due to stigma associated with getting tested for HIV.' (Physician)

Participants in the participatory interview also raised concerns of confidentiality of their HIV status as another factor affecting decisions to report the injury or not:

'Some health workers do not report needle stick injuries due to fear of potentially disclosing their HIV status.' (Hospital matron)

Participants indicated that there are such perceptions that reporting the exposure or injury signals that one is inexperienced, as inexperienced HWs (mainly students) over-report even trivial injuries:

'Yes, we have a reporting system, but students are usually the ones who report needle-stick injuries, hospital staffs seldom do. The root cause of this phenomenon is attributed to perception that students are less experienced and therefore have more accidents.' (Nurse)

Out of the 28 HWs who reported an injury, 14 (50\%) were offered disease testing but only $6(22 \%)$ received PEP. Although nurses suffer fewer injuries compared to other HWs, overall they were keener on reporting on their injuries (73\%) 
TABLE 4: Reporting, counselling, testing and receipt of post exposure prophylaxis after an injury or exposure by job role.

\begin{tabular}{|c|c|c|c|c|c|}
\hline Post-exposure action & Physicians & Nurses & Medical attendants & Total & $\begin{array}{c}\text { Fisher's test } \\
(p \text {-value })\end{array}$ \\
\hline Reporting of injuries or exposure & $(n=10)$ & $(n=19)$ & $(n=17)$ & $(N=46)$ & \multirow{3}{*}{0.273} \\
\hline Yes $n(\%)$ & $6(60)$ & $14(73)$ & $8(12)$ & $28(60)$ & \\
\hline No $n(\%)$ & $4(40)$ & $5(27)$ & $9(88)$ & $18(40)$ & \\
\hline Offered counselling after injury or exposure & $(n=6)$ & $(n=14)$ & $(n=8)$ & $(N=28)$ & \multirow{3}{*}{0.025} \\
\hline Yes $n(\%)$ & $3(50)$ & $1(7)$ & $4(50)$ & $8(25)$ & \\
\hline No $n(\%)$ & $3(50)$ & $13(93)$ & $4(50)$ & $20(75)$ & \\
\hline Offered disease testing after injury or exposure & $(n=6)$ & $(n=14)$ & $(n=8)$ & $(N=28)$ & \multirow{3}{*}{0.798} \\
\hline Yes $n(\%)$ & $3(50)$ & $6(43)$ & $5(62)$ & $14(50)$ & \\
\hline No $n(\%)$ & $3(50)$ & $8(57)$ & $3(38)$ & $14(50)$ & \\
\hline Received PEP & $(n=6)$ & $(n=14)$ & $(n=8)$ & $(N=28)$ & \multirow{3}{*}{0.999} \\
\hline Yes $n(\%)$ & $1(17)$ & $3(12)$ & $2(25)$ & $6(22)$ & \\
\hline No $n(\%)$ & $5(83)$ & $11(78)$ & $6(75)$ & $22(78)$ & \\
\hline
\end{tabular}

PEP, post exposure prophylaxis.

TABLE 5: Availability and accessibility of post exposure prophylaxis services as reported by health workers.

\begin{tabular}{|c|c|c|c|c|c|}
\hline Post-exposure action & Physicians & Nurses & Medical attendants & Total & $\begin{array}{c}\text { Fisher's Test } \\
\text { ( } p \text {-value) }\end{array}$ \\
\hline Availability of PEP guidelines & $(n=28)$ & $(n=124)$ & $(n=84)$ & $(N=236)$ & \multirow[b]{2}{*}{0.98} \\
\hline Yes $n(\%)$ & $19(68)$ & $83(67)$ & $55(65)$ & $157(66)$ & \\
\hline PEP available at no cost & $(n=28)$ & $(n=124)$ & $(n=84)$ & $(N=236)$ & \multirow{3}{*}{0.128} \\
\hline Yes $n(\%)$ & $24(85)$ & $83(67)$ & $56(66)$ & $163(69)$ & \\
\hline No/Don't know $n(\%)$ & $4(15)$ & $41(33)$ & $28(34)$ & $73(31)$ & \\
\hline Yes $n(\%)$ & $22(78)$ & $88(71)$ & $54(64)$ & $164(69)$ & \multirow[t]{2}{*}{0.223} \\
\hline No/Don't know $n(\%)$ & $5(22)$ & $36(29)$ & $30(36)$ & $72(31)$ & \\
\hline Person responsible for PEP & $(n=28)$ & $(n=124)$ & $(n=84)$ & $(N=236)$ & \multirow{3}{*}{0.942} \\
\hline Yes $n(\%)$ & $19(68)$ & $79(64)$ & $55(65)$ & $153(65)$ & \\
\hline No/Don't know $n(\%)$ & $9(32)$ & $45(36)$ & $29(35)$ & $83(35)$ & \\
\hline
\end{tabular}

PEP, post exposure prophylaxis.

than physicians $(60 \%)$ or medical assistants $(12 \%)$. Despite their high reporting tendencies, nurses have a comparatively low chance of being offered counselling $(7 \%)$, disease testing $(43 \%)$ or receiving PEP $(12 \%)$ than their counterparts. Notably, though their reporting tendencies are lower than the other HWs, medical attendants received disease testing (62\%) and PEP (25\%) in higher proportions compared to physicians (disease testing 50\%) (PEP 17\%) (Table 4).

However, qualitative data could not provide details on reasons behind underreporting of splashes and differences in counselling patterns amongst participants.

\section{Post exposure prophylaxis availability and accessibility}

Amongst the KAP respondents, the majority reported that: PEP guidelines were available $(66 \%)$, PEP was being provided at no cost (69\%), PEP was easily accessible (69\%) and that a person responsible for PEP is available at their facility $(65 \%)$ (Table 5).

According to Tanzania National PEP Guidelines (2012), each department or unit in the hospital should have ARV starter kits to ensure that PEP is initiated as soon as possible following an injury. However, observation checklists revealed that across the three hospitals, these important medicines were only available in the hospital pharmacy and dispensing section.

Once HWs are injured or exposed, they report the incident to either the hospital matron or the HW responsible for PEP administration and undergo an assessment of the risk. In the case of night shifts, HWs report the incident to the nurse supervisor or the doctor on call.

Decentralising supply of ARV starter packs for PEP to various hospital sections, such as wards, was deterred by the concern of low shelf life of ARVs provided in the participating hospitals. If provided to units, there are chances of medicines expiring unnoticed. One in-depth interview participant clarified:

'The problem is that we receive ARVs that are close to expiring. So if we keep them in the wards, there is a risk that these drugs might expire without the in-charges of the wards knowing.' (Pharmacist)

\section{Discussion}

This study has described the prevalence and types of occupational exposures, reporting and services offered to exposed HWs in the three hospitals of the Tanzania's lake zone. We found that nearly one third of respondents had experienced percutaneous exposures (NSIs and 
sharp injuries) and one fifth had been exposed to splashes. However, when disaggregated, the prevalence of NSIs, sharps injuries and splashes was found to be lower compared to studies done in Kenya and Ethiopia (Mbaisi et al. 2013; Tebeje \& Hailu 2010). The presence of percutaneous exposures in this study could be attributed to the fact that being public hospitals (SRH and $\mathrm{MRH}$ ) and public or private hospital (SDDH) with low staffing levels, the three hospitals have a high patient load per HW, a factor that was reported to be associated with occupational injuries in a previous study (Rapiti et al. 2005). In addition, the high rate of splashes compared to NSIs and sharp injuries is likely due to the fact that the majority of respondents were from the labour and delivery wards, a finding which is similar to a study done in Ethiopia (Tebeje \& Hailu 2010). However, it would be worth monitoring this indicator closely (ward or department in which the HW works) during project implementation to get a better understanding.

The risk of occupational exposure to HIV depends on the activities and duties performed by the HW. In this study, physicians had higher numbers of occupational exposures compared to other HWs. Unlike findings from the previous studies (Mbaisi et al. 2013; Tebeje \& Hailu 2010) which showed nurses having a higher risk of occupational exposure due the fact that they were more likely to have more contact with patients, physicians do perform more invasive procedures which expose them to contact with body fluids than nurses. Therefore, physicians working in the three hospitals could benefit from continuous training and reliable supply of safety engineered devices (SEDs) and PPEs.

In this study, very few (28\%) of splash exposures were reported compared to needle sticks (80\%) and sharps injuries (68\%). This is consistent with a previous study (Kessler et al. 2011) which showed that whilst splashes were reported by only $18.1 \%$ of one hundred and five participants, $66 \%$ of the 103 participants who sustained sharp injuries reported their injury. This might indicate perceptions that splash exposures are not as serious as needle sticks or sharps injuries and lead HWs to minimise the risk of infection from splashes since the chance of becoming infected with HIV, HBV or HCV from the splash injury is one third the chance after a needle-stick or sharps injury (Delisio 2012) or that HWs do not follow existing protocols for reporting and treatment as stated in the national guidelines (NACP 2012). Additionally, a previous research showed that although acquiring HIV after a splash injury is $0.09 \%$, the risk is greater if the injury is prolonged (Wines et al. 2008).

One finding that could be of interest is that the majority of nurses reported the exposure though they were least likely to experience an occupational exposure and they had a comparatively low chance of being offered counselling, disease testing or receiving PEP than their counterparts.
Furthermore, although more physicians experienced and reported the exposures as compared to others, few of them received PEP medicines. However, this finding contradicts the finding of the study in Switzerland (Voide et al. 2012) which reported that $42.7 \%$ of doctors performing invasive procedures under-reported needle stick and sharp injuries (NSSIs). The consequences of not reporting nor using PEP medicines as required might culminate in a hidden increase in occupational acquired infections in other types of HWs. Specific interventions to emphasize the importance of reporting every occupational exposure might address this gap during the implementation of the HWS programme. In addition, all job roles at risk of injury or exposure should receive adequate and (when) necessary targeted training.

Counselling and PEP use after occupational exposure or injury was minimal in this study though half were offered disease testing. The reasons behind these differences could not be determined as the study design did not allow for follow up of unique responses identified upon data analysis (Creswell \& Plano Clark 2011). In the three hospitals, PEP medicines are available at outpatient dispensing units and the main hospital pharmacy, a practice that contradicts the national guidelines and may delay early initiation of ARVs for PEP as recommended by the national guidelines (NACP 2012). In addition, it was clearly pointed out during interviews that HIV stigma continues to deter HWs from accessing PEP services from restricted locations. This finding is similar to an Ethiopian study which reported lack of awareness of the existence of PEP services and stigma related to HIV and/or AIDS as the main reasons for not accessing PEP following an occupational injury (Tebeje \& Hailu 2010).

\section{Limitations of the study}

Although this study was set to generate important evidence with regards to determining baseline information on prevalence and types of occupational injuries, reporting and services offered after the exposure, there are some limitations that need to be acknowledged.

The most obvious limitation was its cross-sectional nature that limits making firm conclusions about causal relationships between variables. Therefore, relationships amongst variables were interpreted with caution. Response bias was another limitation as data was collected through interviews and questionnaires and could not be verified through other records such as an occupational injury registry and PEP forms. In addition, a number of respondents who were interviewed did not answer some questions leading to nonresponse bias. These limitations however, were addressed by data triangulation through the use of multiple tools and methodological triangulation by use of a mixed method approach. 


\section{Conclusion}

Occupational exposures are common in the three hospitals in Tanzania's lake zone. HWs in these hospitals underreport percutaneous exposures and splashes are rarely reported. Although PEP medicines are available at no cost in these hospitals, counselling and use of PEP services is still suboptimal. During HWS programme implementation, specific interventions should establish a functional system for reporting of occupational exposures, ensure that counselling and testing for HIV are offered to all exposed HWs and when indicated, encourage use of PEP medicines after occupational exposures. PEP starter packs should therefore be available and known to HWs in each ward and or department. A system should exist to regularly check expiry dates and have backup starter kits on hand. Future research should establish factors that determine compliance to PEP procedures.

\section{Acknowledgements}

Ella Gudwin, Francisco Chibunda, N'Deye Niang, Steven Tilubuza and Elikem Tomety Archer contributed to the overall HWS programme baseline evaluation from which this article originates. We also thank hospital officials, HWs and other stakeholders for participating in the study. Furthermore, we are thankful to our research assistants for assisting us in the data collection. A special thanks goes to our mentor, Dr Edward de Vos, for his helpful critique of the manuscript.

\section{Competing interests}

The authors declare that they have no financial or personal relationship(s) that may have inappropriately influenced them in writing this article.

\section{Authors' contributions}

R.K. (Bugando Medical Centre) designed the study and study tools, collected the data, drafted, revised and approved the final manuscript. A.K. (St. Francis University College of Health and Allied Sciences) designed the study and study tools, collected the data, carried out the data analysis, drafted, revised and approved the final manuscript. G.F. (AmeriCares) carried out the data analysis, drafted, revised and approved the final manuscript. S.B. (AmeriCares) designed the study and study tools, drafted, revised and approved the final manuscript

\section{References}

Birdsall, K., Hajiyiannis, H., Parker, W. \& Nkosi, Z., 2004, 'Post-exposure prophylaxis (PEP) in South Africa: Analysis of calls to the national AIDS helpline', viewed 19 April 2015, from http://www.cadre.org.za/node/172.

Cardo, D.M., Culver, D.H., Ciesielski, C.A., Srivastava, P.U., Marcus, R., Abiteboul, D. et al., 1997, 'A case-control study of HIV seroconversion in health care workers after percutaneous exposure', New England Journal of Medicine 337(21), 1485-1490. percutaneous exposure', New England Journal of Medicine 337(21),
PMID: 9366579, http://dx.doi.org/10.1056/NEJM199711203372101

Creswell, J.W., Klassen, A.C., Plano Clark, V.L. \& Clegg Smith, K., 2011, Best practices for mixed methods research in the health sciences, Office of Behavioral and Social Sciences Research.
Creswell, J.W. \& Plano Clark, V.L., 2011, Designing and conducting mixed methods research, 2 nd edn., Sage Publications, Thousand Oaks.

Delisio, N., 2012, 'Bloodborne infection from sharps and mucocutaneous exposure: A continuing problem', American Nurse Today.

Feilzer, M.Y., 2010, 'Doing mixed methods research pragmatically: Implications for the rediscovery of pragmatism as a research paradigm', Journal of Mixed Methods Research 4(1), 6-16. http://dx.doi.org/10.1177/1558689809349691

Gale, N.K., Heath, G., Cameron, E., Rashid, S. \& Redwood, S., 2013, 'Using the framework method for the analysis of qualitative data in multi-disciplinary health research', BMC Medical Research Methodology, 13(1), 117. PMID: 24047204 http://dx.doi.org/10.1186/1471-2288-13-117

Geer, L.A., Curbow, B.A., Anna, D.H., Lees, P.S.J. \& Buckley, T.J., 2006, 'Development of a questionnaire to assess worker knowledge, attitudes and perceptions underlying dermal exposure', Scandinavian Journal of Work, Environment \& Health 32(3), 209-218. PMID: 16804624.

Gold, J. \& Tomkins, M., 2005, 'Occupational post-exposure prophylaxis for HIV: A discussion paper', paper presented at the Technical Meeting for the Development
of Guidelines and Policies on Occupational and Non-Occupational Post Exposure of Guidelines and Policies on Occupational and Non-Occupational Post Exposure
Prophylaxis (PEP), World Health Organization/International Labour Organization, Prophylaxis (PEP), Word
Geneva, Switzerland.

Kessler, C.S., McGuinn, M., Spec, A., Christensen, J., Baragi, R. \& Hershow, R.C., 2011 'Underreporting of blood and body fluid exposures among health care students and trainees in the acute care setting: A 2007 survey', American Journal of and trainees in the acute care setting: A 2007 survey', American Journal of
Infection Control 39(2), 129-134. PMID: 21356431, http://dx.doi.org/10.1016/j. Infection Control
ajic.2010.06.023

Kuhar, D.T., Henderson, D.K., Struble, K.A., Heneine, W., Thomas, V., Cheever, L.W. et al., 2013, 'Updated US public health service guidelines for the management of al., 2013, "Updated US public health service guidelines for the management of for postexposure prophylaxis', Infection Control and Hospital Epidemiology 34(9), for postexposure prophylaxis', Infection Control and Hospital Epide

Kwesigabo, G., Mwangu, M.A., Kakoko, D.C., Warriner, I., Mkony, C.A., Killewo, J. et al., 2012, 'Tanzania's health system and workforce crisis', Journal of Public Health Policy 33(S1), S35-S44. PMID: 23254848, http://dx.doi.org/10.1057/jphp.2012.55

Lee, R., 2009, 'Occupational transmission of bloodborne diseases to healthcare workers in developing countries: Meeting the challenges', The Journal of Hospital Infection 72(4), 285-291. PMID: 19443081, http://dx.doi.org/10.1016/j. jhin.2009.03.016

Mbaisi, E.M., Ng'ang'a, Z., Wanzala, P. \& Omolo, J., 2013, 'Prevalence and factors associated with percutaneous injuries and splash exposures among health-care workers in a provincial hospital, Kenya, 2010', Pan African Medical Journal 14(1), 10. PMID: 23504245, http://dx.doi.org/10.11604/pamj.2013.14.10.1373

NACP, 2012, National Guidelines for the clinical management of HIV and AIDS, 3rd edn., United Republic of Tanzania.

Prüss-Üstün, A. \& Rapiti, E., 2003, Sharps injuries: Global burden of disease from sharps injuries to health-care workers, WHO.

Prüss-Üstün, A., Rapiti, E. \& Hutin, Y., 2005, 'Estimation of the global burden of disease attributable to contaminated sharps injuries among health-care workers', American Journal of Industrial Medicine 48(6), 482-490. PMID: 16299710.

Rapiti, E., Prüss-Üstün, A. \& Hutin, Y., 2005, Sharps injuries: Assessing the burden of disease from sharps injuries to health-care workers at national and local levels, WHO.

Sagoe-Moses, C., Pearson, R.D., Perry, J. \& Jagger, J., 2001, 'Risks to health care workers in developing countries', The New England Journal of Medicine 345(7), 538-541.

Sari, S.Y.I., Ibrahim, K., Haroen, H., Afriandi, I., Sunjaya, D.K., Hinduan, Z.R. et al., 2011, 'Knowledge, attitude and perceived adherence with universal precautions among health care workers in the obstetrics and gynaecology department of an Indonesian teaching hospital', International Journal of Infection Control 7(4). http://dx.doi.org/10.3396/ijic.V7i4.036.11

Sultan, B., Benn, P. \& Waters, L., 2014, 'Current perspectives in HIV post-exposure prophylaxis', HIV/AIDS (Auckland, N.Z.) 6, 147-158. PMID: 25368534, http:// dx.doi.org/10.2147/HIV.S46585

TACAIDS, 2013, Tanzania HIV/AIDS and Malaria Indicator Survey 2011-2012, Tanzania Commission for AIDS (TACAIDS), Zanzibar AIDS Commission (ZAC), National Bureau of Statistics (NBS), Office of the Chief Government Statistician (OCGS) and ICF International.

Tebeje, B. \& Hailu, C., 2010, 'Assessment of HIV post-exposure prophylaxis use among health workers of governmental health institutions in Jimma Zone, Oromiya Region, Southwest Ethiopia', Ethiopian Journal of Health Sciences 20(1), 55-64. PMID: 22434961.

Varghese, G.M., Abraham, O. \& Mathai, D., 2003, 'Post-exposure prophylaxis for blood borne viral infections in healthcare workers', Postgraduate Medical Journal 79(932), 324-328. PMID: 12840120.

Voide, C., Darling, K.E.A., Kenfak-Foguena, A., Erard, V., Cavassini, M. \& Lazor-Blanchet, C., 2012, 'Underreporting of needlestick and sharps injuries among healthcare workers in a Swiss University Hospital', Swiss Medical Weekly 142, w13523. PMID: 22328010, http://dx.doi.org/10.4414/smw.2012.13523

WHO, 2015, 'Post-exposure prophylaxis (PEP)', viewed 19 April 2015, from http:// www.who.int/hiv/topics/prophylaxis/en/

Wines, M.P., Lamb, A., Argyropoulos, A.N., Caviezel, A., Gannicliffe, C. \& Tolley, D., 2008, 'Blood splash injury: An underestimated risk in endourology', Journal of Endourology 22(6), 1183-1188. PMID: 18498231, http://dx.doi.org/10.1089/ end.2008.0052 